The Astrophysical Journal, 480:481-491, 1997 May 10

(C) 1997. The American Astronomical Society. All rights reserved. Printed in U.S.A.

\title{
THE PROTOGALACTIC ORIGIN FOR COSMIC MAGNETIC FIELDS
}

\author{
Russell M. Kulsrud, ${ }^{1}$ Renyue Cen, ${ }^{1}$ Jeremiah P. Ostriker, ${ }^{1}$ AND Dongsu Ryu ${ }^{2}$ \\ Received 1996 July 29; accepted 1996 December 5
}

\begin{abstract}
It is demonstrated that strong magnetic fields are produced from a zero initial magnetic field during the pregalactic era, when the galaxy is first forming. Their development proceeds in three phases. In the first phase, weak magnetic fields are created by the Biermann battery mechanism. During the second phase, results from a numerical simulation make it appear likely that homogenous isotropic Kolmogorov turbulence develops that is associated with gravitational structure formation of galaxies. Assuming that this turbulence is real, then these weak magnetic fields will be amplified to strong magnetic fields by this Kolmogorov turbulence. During this second phase, the magnetic fields reach saturation with the turbulent power, but they are coherent only on the scale of the smallest eddy. During the third phase, which follows this saturation, it is expected that the magnetic field strength will increase to equipartition with the turbulent energy and that the coherence length of the magnetic fields will increase to the scale of the largest turbulent eddy, comparable to the scale of the entire galaxy. The resulting magnetic field represents a galactic magnetic field of primordial origin. No further dynamo action after the galaxy forms is necessary to explain the origin of magnetic fields. However, the magnetic field will certainly be altered by dynamo action once the galaxy and the galactic disk have formed.

It is first shown by direct numerical simulations that thermoelectric currents associated with the Biermann battery build the field up from zero to $10^{-21} \mathrm{G}$ in the regions about to collapse into galaxies, by $z \sim 3$. For weak fields, in the absence of dissipation, the cyclotron frequency $-\omega_{\mathrm{cyc}}=e \boldsymbol{B} / m_{\mathrm{H}} c$ and $\omega /(1+\chi)$, where $\omega=\nabla \times v$ is the vorticity and $\chi$ is the degree of ionization, satisfy the same equations, and initial conditions $\omega_{\text {cyc }}=\omega=0$, so that, globally, $-\omega_{\text {cyc }}(r, t)=\omega(r, t) /(1+\chi)$. The vorticity grows rapidly after caustics (extreme nonlinearities) develop in the cosmic fluid. At this time, it is made plausible that turbulence has developed into Kolmogorov turbulence. Numerical simulations do not yet have the resolution to demonstrate that, during the second phase, the magnetic fields are amplified by the dynamo action of the turbulence. Instead, an analytic theory of the turbulent amplification of magnetic fields is employed to explore this phase of the magnetic field development. From this theory, it is shown that, assuming the turbulence is really Kolmogorov turbulence, the dynamo action of this protogalactic turbulence is able to amplify the magnetic fields by such a large factor during the collapse of the protogalaxy that the power into the magnetic field must reach saturation with the turbulent power. For the third phase, there is as yet no analytic theory capable of describing this phase. However, preliminary turbulence calculations currently in progress seem to confirm that the magnetic fields may proceed to equipartition with the turbulent energy, and that the coherence length may increase to the largest scales. Simple physical arguments are presented that show that this may be the case. Such an equipartition field is actually too strong to allow immediate collapse to a disk. Possible ways around this difficulty are discussed.
\end{abstract}

Subject headings: galaxies: evolution - ISM: magnetic fields - methods: numerical — MHD turbulence

\section{INTRODUCTION}

How do cosmic magnetic fields arise? It has been the general belief that there is no natural mechanism to create a strong primordial magnetic field prior to the formation of galaxies (e.g., see Ruzmaikin, Shukurov, \& Sokoloff 1988) For this reason, attention has been concentrated on the production of galactic fields after the galactic disks formed. It is assumed that some weak field, of order $10^{-17} \mathrm{G}$, is initially present at the time of the beginning of the galactic disk, and this weak field is amplified to its present strength of a few microgauss by dynamo action of interstellar hydro-

\footnotetext{
${ }^{1}$ Princeton University Observatory, Peyton Hall, Princeton, NJ 08544-1001; rkulsrud@astro.princeton.edu, cen@astro.princeton.edu, jpo@astro.princeton.edu.

${ }^{2}$ Department of Astronomy and Space Science, Chungnam National University, Daejeon, 305-764, Korea; ryu@sirius.chungnam.ac.kr.
}

dynamic turbulence (for a survey of the galactic dynamo theory, see Ruzmaikin et al. 1988). But the theory for this amplification suffers from the problem of rapid amplification of small-scale fields that inhibit the amplification of large-scale fields (Kulsrud \& Anderson 1992). In addition, the origin of the weak "seed field" is still open.

Because of these difficulties, it is of interest to reexamine the possibility that a strong primordial magnetic field can be produced in the pregalactic era. We have done that in this paper, and we come to the following picture of the magnetic field origin.

Prior to the formation of galaxies, the universe is by no means uniform. Indeed, it is now known that small relative density perturbations, $\delta \rho / \rho$, present at the time of recombination, grow to finite amplitude and form the present galaxies, clusters of galaxies, and other structures, at a variety of epochs. When $\delta \rho / \rho \approx 0.1$, shocks form, and the resulting heated, electrically conducting fluid produces electric cur- 
rents that generate magnetic fields even from field-free initial conditions. Such fields, which are quite weak $\left(\approx 10^{-21} \mathrm{G}\right)$, would, after compression into the galactic disk, produce the required seed field for the galactic dynamo.

However, this is not the end of the story. The shocks also generate vorticity on all scales. This vorticity is strong enough that its vortex cells turn over and generate Kolmogorov turbulence. The energy spectra of this turbulence has the well-known power law and extends down to the viscous scale. (It is true that the medium is compressible. However, the shear motions in the turbulence are themselves incompressible. This is because the difference in frequencies between compressible and shear motions do not permit the sound waves to interact strongly with the incompressible shear motions. We can thus regard the turbulence as incompressible and of the nature considered by Kolmogorov.)

The eddies in the Kolmogorov turbulence turn over at a rate that is fastest for the smallest eddy. This smallest eddy amplifies the magnetic field at a rate comparable to its turnover rate. The existence of a Kolmogorov cascade from large eddies to small eddies is crucial because it enables the slowly rotating large eddies to drive rapidly rotating small eddies, which rapidly amplify the magnetic field.

It will be shown that conditions are always such that during the collapse of a protogalaxy, the smallest eddy turns over several hundred times. This would lead to an increase of the magnetic field energy by a corresponding number of powers of $e$, the base of natural logarithms, if saturation processes did not set in. Instead, the magnetic field saturates and comes into equipartition with the pregalactic turbulence. Such a saturated field is of sufficient strength to provide a primordial origin for galactic magnetic fields.

If the dynamics of the magnetic field played no role, then the magnetic field would be very chaotic on small scales and the magnetic energy could be concentrated on scales smaller than the smallest eddy. However, as the field strengthens, the magnetic field on these smallest scales resists amplification, and the total magnetic field continues to strengthen on scales comparable to that of the smallest hydrodynamic turbulent eddy only. But later, when the field becomes even stronger, the smallest hydrodynamic eddies become suppressed because of the increasing drain of energy to the magnetic field. As a result, the spectrum of the turbulence becomes truncated at larger scales, and the scale of the smallest hydrodynamic eddy increases. Eventually, only the largest eddies survive. During these later stages of saturation, only the magnetic energy at the largest scales is amplified, and the magnetic field eventually becomes coherent on these largest scales.

Thus, during the pregalactic era, the magnetic field goes through three phases. During the first phase, thermoelectric currents generated by shocks increase the magnetic field strength to a value of order $10^{-21} \mathrm{G}$. This process is known as the Biermann battery mechanism, after its discoverer (Biermann 1950). When this level of magnetic field strength is reached, the dynamo action of the turbulence becomes faster than the battery action, and the second phase commences. During this phase, the turbulence forms a Kolmogorov spectrum down to the viscous scale. The smallest eddy at this scale does all the work in amplifying the magnetic field and continues to amplify it until saturation sets in. Finally, as saturation is approached, the third phase takes over. During this third phase, the magnetic field approaches equipartition with the hydrodynamic turbulence and becomes coherent on very large scales.

To examine these three phases, we modified a standard code for the numerical simulation of the gravitational structure (Ryu et al. 1993). We first added the magnetic differential equation to the standard simulation equations in order to study the creation and evolution of the magnetic field, starting from a zero initial field. (Because the magnetic field is at first very weak, we neglected any magnetic forces in the numerical simulation, so the basic numerical solution for velocities and pressures was unaffected.) We found that the hydrodynamic turbulence does indeed create a magnetic field, increasing it to a field strength of about $10^{-21} \mathrm{G}$, as expected for the first phase. However, the numerically simulated magnetic field did not increase beyond this point. The reason is that only a small part of the Kolmogorov turbulence was captured in the numerical simulation. The smaller eddies, which would be the most important for dynamo amplification, were suppressed by numerical resolution. The really important eddies (those on the smallest scale) were on a scale smaller than the grid size and not seen at all. The only eddies captured in the numerical simulation turn over at too slow a rate to do more than overcome the damping of the field by numerical resistivity. Thus, the simulation has too low a resolution to correctly represent the second phase of dynamo amplification.

Therefore, the remaining conclusions concerning the enormous amplification of the magnetic field strength expected for the second phase cannot be simulated until the numerical resolution is improved. To properly examine this amplification, we must employ an analytical method. To do this, we assume that the Kolmogorov spectrum inferred from the numerical simulation is real. This is plausible since the numerical evidence for the Kolmogorov spectrum makes this assumption reasonable. The large-scale eddies found in the simulations actually do turn over a sufficient number of times to produce a Kolmogorov spectrum. Furthermore, in the numerical simulations, where an approximately $-5 / 3$ power law was found over a limited range in wavenumber space, this range became larger when the numerical resolution was improved. Since the ideas of Kolmogorov turbulence are now generally accepted, we felt secure in extrapolating the spectrum to the smallest viscous scales.

Thus, assuming that the Kolmogorov spectrum of turbulence is present during phase two, we employed a standard theory for the dynamo amplification of magnetic energy by hydrodynamic turbulence in the weak field limit (Kulsrud \& Anderson 1992). To evaluate the total amount of amplification, we first scaled the total turbulent energy to the thermal energy. From the numerical simulations, we know that these energies are comparable. Next, to find the scale of the smallest eddy, we needed the plasma temperature and density. We normalized the temperature to the Jeans's temperature. This gives a good approximation to the actual temperature during the time of the collapse of the protogalaxy. Using these results, we are able to make an estimate of the number of amplifications of the magnetic energy. We find that this estimate only depends on the normalization constants, which are of order unity, and on the inverse square root of the galactic mass. For a baryonic mass in the range of a galactic mass, this estimate for the number of $e$-foldings of the magnetic energy is extremely large and 
demonstrates that the magnetic field will probably reach saturation with the turbulence.

Our examination of the third phase, during which the magnetic field is strong and drives itself to coherence on a large scale, is still in progress (Chandran 1996). However, physical arguments can be made that support the conclusion that the magnetic field should become coherent on a scale comparable to the size of the galaxy.

Therefore, on the basis of the above considerations and calculations, we argue that the origin of galactic fields is primordial, i.e., predates the galaxies in their equilibrium state. Even if no magnetic field were present at the time of recombination, a magnetic field would be generated by the Biermann battery mechanism driven by the gravitational structure formation. Subsequently, during gravitational collapse of the protogalaxy to the galaxy, the residual turbulence will drive dynamo action that will increase the magnetic field strength to saturation. Finally, the strong magnetic field will smooth itself out on very large scales.

The plan of the paper is as follows. In $\S 2$, we describe the numerical simulation that shows the initial generation of the magnetic field, during the first phase, by the Biermann battery mechanism. In $\S 3$, we apply the analytic theory of Kulsrud \& Anderson (1992) to calculate the amount of dynamo amplification during the second phase. We show that this amplification is more than adequate to make the magnetic field sufficiently strong so that the power into it reaches saturation with the hydrodynamic turbulence power. In $\S 4$, we present the physical arguments that imply that during the third phase, the magnetic field becomes coherent on large scales. In $\S 5$, we present our conclusions.

\section{NUMERICAL SIMULATION OF INITIAL GROWTH OF MAGNETIC FIELD}

The first phase of the evolution of the protogalactic magnetic field can be simulated numerically by modifying a cosmological hydrodynamic code that is normally used to simulate structure formation (e.g., Ryu et al. 1993). During this phase, the magnetic field is too weak to be of dynamic significance during the simulation. Thus, we include the magnetic field passively in the code because it does not affect the motions of the plasma. In the other words, we add the evolution equation for the magnetic field

$$
\frac{\partial B}{\partial t}=\nabla \times(v \times B)
$$

to the code, where $v$ is obtained from the hydrodynamic part of the code without any magnetic forces. Thus, $\boldsymbol{B}$ can be followed easily without disturbing the rest of the simulation.

The question then arises concerning what initial value to take for the magnetic field in the modified simulation. According to equation (1), it must be initially nonzero if $\boldsymbol{B}$ is to be nonzero in time. A number of proposals have been made for the generation of a small initial magnetic field in the early universe. One of the more promising of these is the Biermann battery mechanism, which makes use of an extra pressure gradient term in Ohm's law (Biermann 1950). Employing this mechanism, we start with zero field at the beginning of the simulation and include an extra term in the differential equation for the magnetic field. Thus, equation (1) is replaced by

$$
\frac{\partial \boldsymbol{B}}{\partial t}=\nabla \times(\boldsymbol{v} \times \boldsymbol{B})+\frac{c \nabla p_{e} \times \nabla n_{e}}{n_{e}^{2} e},
$$

where $n_{e}$ is the electron density and $p_{e}$ is the electron pressure.

If the ionization fraction, $\chi$, is taken constant in space, and the electron temperature is taken equal to the neutral temperature, then $n_{e} / n_{B}(1+\chi)=p_{e} / p$. Here $n_{B}$ is the baryonic number density. From this result, equation (2) reduces to

$$
\frac{\partial \boldsymbol{B}}{\partial t}=\nabla \times(v \times B)+\frac{\nabla p \times \nabla \rho}{\rho^{2}} \frac{c m_{\mathrm{H}}}{e} \frac{1}{1+\chi},
$$

where $m_{\mathrm{H}}$ is the hydrogen mass; $\rho=n_{B} M /(1+\chi)$ for a hydrogen gas. Equation (3) involves only the quantities that can be supplied from the hydrodynamic part of the code, so it is easy to incorporate the solution of equation (3) into the cosmological hydrodynamic code and to follow the evolution of $\boldsymbol{B}$ in time as the simulation proceeds.

For any barytropic flow $[p \equiv p(\rho)]$, the last source term is zero because $\nabla p$ is parallel to $\nabla \rho$. However, in general, for a real fluid in which curved shocks and photoheating can occur, $\nabla p \times \nabla \rho \neq 0$.

Multiplying equation (3) by $e / m_{\mathrm{H}} c$, we get the equation for the cyclotron frequency $\omega_{\mathrm{cyc}}=e \boldsymbol{B} / m_{\mathrm{H}} c$,

$$
\frac{\partial \omega_{\mathrm{cyc}}}{\partial t}=\nabla \times\left(v \times \omega_{\mathrm{cyc}}\right)+\frac{\nabla p \times \nabla \rho}{\rho^{2}} \frac{1}{1+\chi}+\frac{\eta c}{4 \pi} \nabla^{2} \omega_{\mathrm{cyc}} .
$$

We have added a term to represent any resistive diffusion that may be present.

Note that the ionization fraction enters into equation (3) through $1+\chi$, so even a very low ionization fraction is enough to generate magnetic fields. It is only necessary that there be enough electrons to carry the required current with a drift velocity relative to the ions $v_{D}$ less than their thermal velocity $v_{e}$, i.e., $B / 4 \pi L=n_{e} e v_{D} / c<n_{e} e v_{e} / c$, where $L$ is the scale size of the variation of the magnetic field. This condition is satisfied by a large margin in the numerical simulations. (If this condition were not satisfied, an anomalously large resistance would develop because of plasma instabilities, and the magnetic field would be inhibited from growing.)

Equation (3) was incorporated into a numerical simulation for the large-scale structure formation in a standard cold dark matter (CDM) model universe with total $\Omega=1$. The simulation was done in a periodic box with $\left(32 h^{-1}\right.$ $\mathrm{Mpc})^{3}$ volume using $128^{3}$ cells and $64^{3}$ particles from $z_{i}=$ 20 to $z_{\mathrm{f}}=0$. The values of other parameters used are $\Omega_{b}=$ $0.06, h=\frac{1}{2}$, and $\sigma_{8}=1.05$. For the initial condition, we adopted the standard CDM power spectrum with $n=1$, which is modified by the transfer function given by Bardeen et al. (1986). Thus, the simulation is basically the same as that reported in Kang et al. (1994), except that a smaller box, and a smaller number of cells and particles, was used.

The actual equation for the magnetic field in comoving coordinates, which we solved, is

$$
\begin{aligned}
\frac{\partial \boldsymbol{B}}{\partial t}= & \frac{1}{a} \nabla \times(v \times \boldsymbol{B})-2 \frac{\dot{a}}{a} \boldsymbol{B} \\
& -\frac{1}{B_{0} t_{G}} \frac{m_{\mathrm{H}} c}{e} \frac{1}{1+\chi} \frac{1}{a^{2}} \frac{\nabla \rho \times \nabla p}{\rho^{2}},
\end{aligned}
$$


where $B_{0}$ and $t_{G}$ are the normalization constants for the magnetic field and time. This equation was solved by using the total variation diminishing (TVD) scheme (Harten 1983). This is the same scheme as that employed for the solution of the hydrodynamic equations (Ryu et al. 1993), so the accuracy was comparable. The constraint $\boldsymbol{\nabla} \cdot \boldsymbol{B}=0$ was enforced separately in every time step. We assumed $\chi=1$.

In Figure 1, the temporal evolution of the resulting magnetic field is plotted. The upper panel shows the volumeaveraged (solid line) and mass-averaged (dotted line) magnetic energy density $\left(B^{2} / 8 \pi\right)$ as a function of $z$. The lower panel shows the volume-averaged (solid line) and mass-averaged (dotted line) magnetic field strength $(B)$. Note that $B \propto h$, and $h=\frac{1}{2}$ was used. The magnetic field strength at first grows monotonically to the mass-averaged value of order $10^{-21} \mathrm{G}$ by $z \sim 2-3$. In the core of clusters, it reached $10^{-20} \mathrm{G}$ by $z \sim 3$. After this time, the value of the averaged field strength leveled off without further increase. It is believed that the saturation is due to the finite numerical resistivity inherent in the numerical scheme used to solve equation (5).

The contours of the resulting baryonic density $(\rho)$ and magnetic field strength $(B)$ at $z=2$ are shown in Figures 2 and 3. The slice shown has a thickness of $2 h^{-1} \mathrm{Mpc}$ (or 8 cells). The upper panels show the whole region of $32 \times 32$ $h^{-1} \mathrm{Mpc}$, while the lower panels show a magnified region of $10 \times 10 h^{-1} \mathrm{Mpc}$. In Figure 2, the regions with density
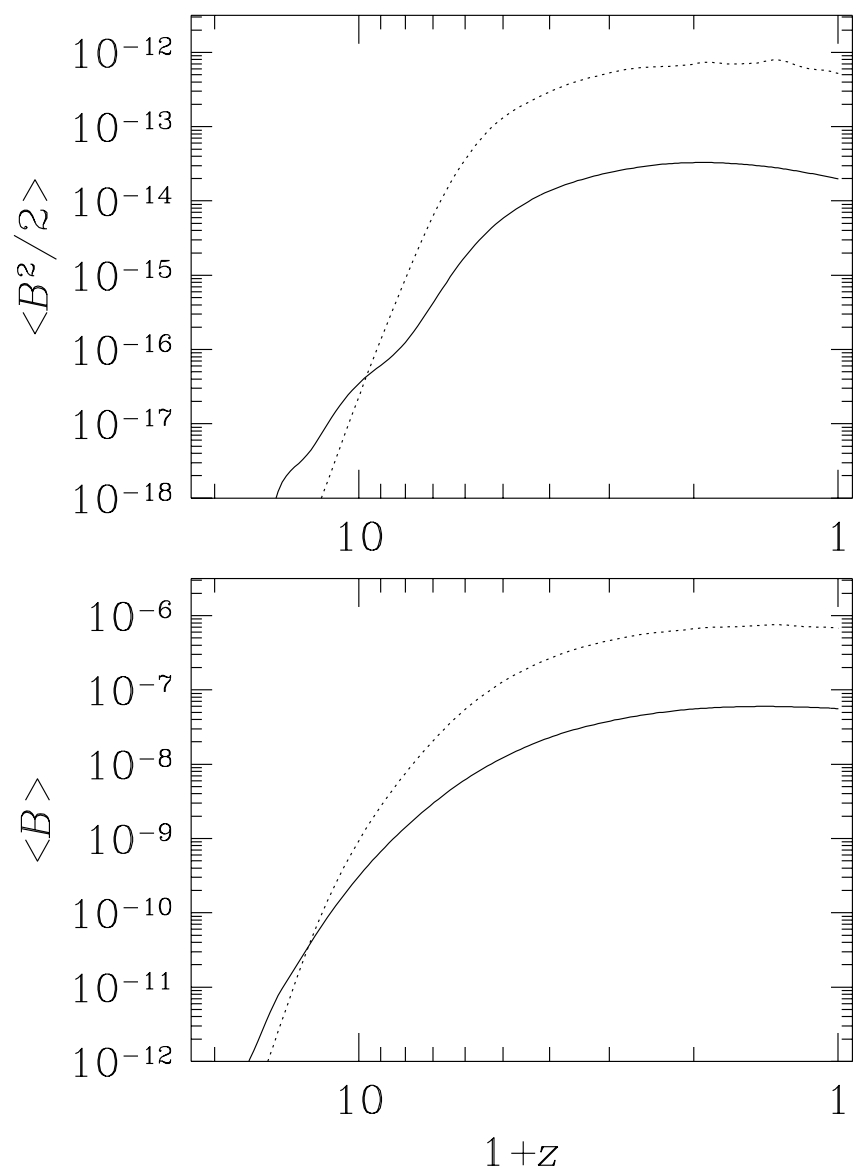

Fig. 1.-Temporal evolution of the magnetic field. The upper panel shows the volume-averaged (solid line) and mass-averaged (dotted line) magnetic energy density $\left(B^{2} / 8 \pi\right)$ as a function of $z$. The lower panel shows the volume-averaged (solid line) and mass-averaged (dotted line) magnetic field strength $(B)$.
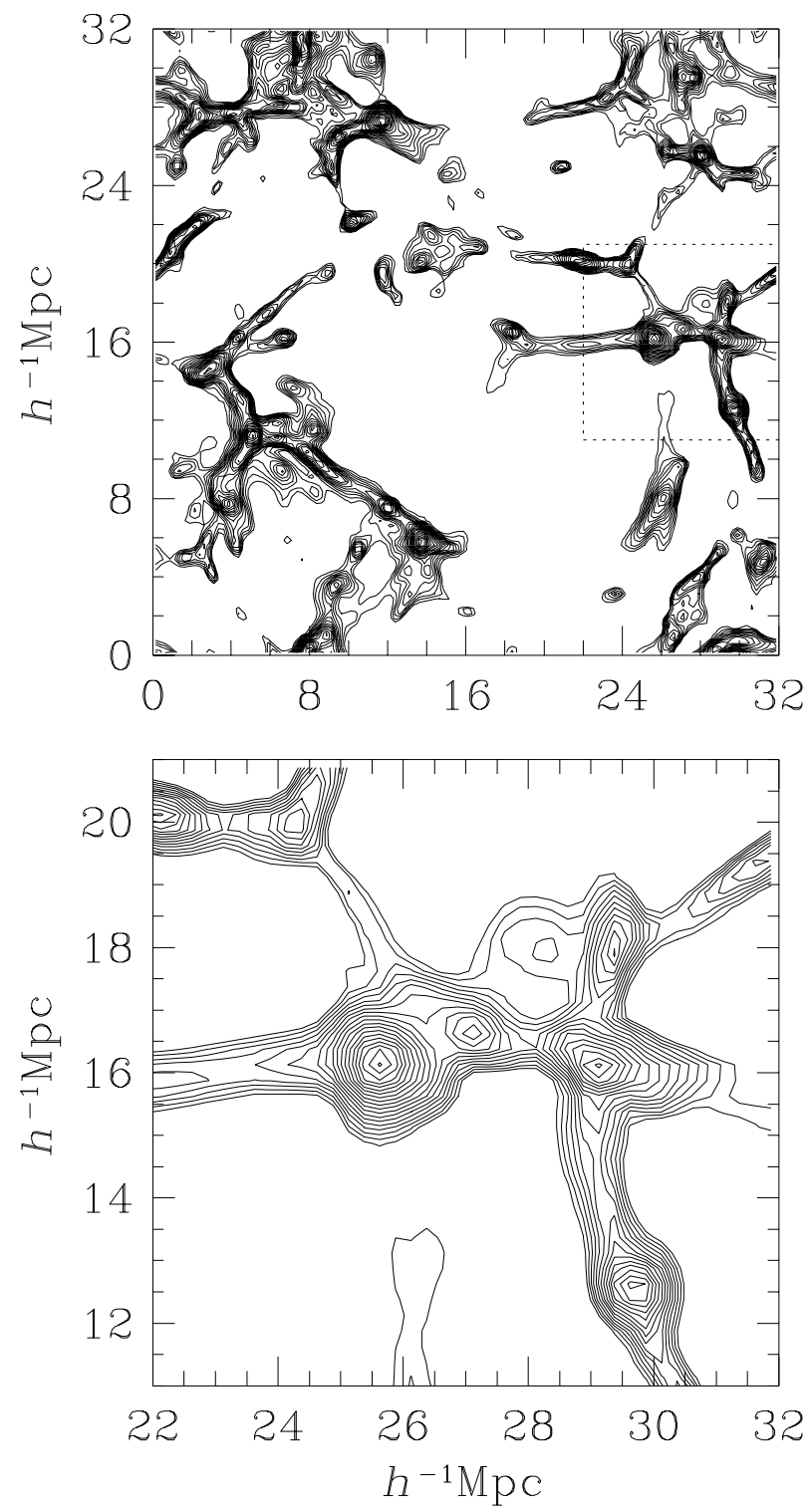

FIG. 2.-Density contours of a slice with a thickness of $2 h^{-1} \mathrm{Mpc}$ (or 8 cells) at $z=2$. The contour lines with density higher than $0.06 \bar{\rho}$ are shown with levels $0.06 \times 10^{k}$ and $k=0,0.1,0.2, \ldots, 2$. The upper panel shows the whole region of $32 \times 32 h^{-1} \mathrm{Mpc}$, while the lower panel shows the magnified region of $10 \times 10 h^{-1} \mathrm{Mpc}$.

higher than the volume-averaged value $(0.06 \bar{\rho})$ are contoured with contour levels $(0.06 \bar{\rho}) \times 10^{k}$ and $k=0$, $0.1,0.2, \ldots, 2$. Similarly, in Figure 3, the regions with magnetic field strength higher than the volume-averaged value $\left(8 \times 10^{-23} \mathrm{G}\right)$ are contoured with contour levels $\left(8 \times 10^{-23}\right.$ G) $\times 10^{k}$ and $k=0,0.1,0.2, \ldots, 2$. As expected, they are very well correlated. Since the magnetic field was mostly generated in the accretion shocks around the clusters, the high-density core regions of the clusters have the strongest magnetic fields.

It is interesting to compare the maximum rms value of $B$, or, alternatively, $\omega_{\text {cyc }}=10^{4} B$, with the same rms mean for the vorticity, $\omega=\nabla \times v$, where $v$ is the fluid velocity. The maximum rms value for $\omega$ is $\sim 10^{-16} \mathrm{~s}^{-1}$ around the clusters in the numerical simulation. This is equal to the cyclotron frequency of an ion in a magnetic field of $\sim 10^{-20} \mathrm{G}$. In other words, $e B / m_{\mathrm{H}} c \approx \omega$ in the clusters. This is not sur- 

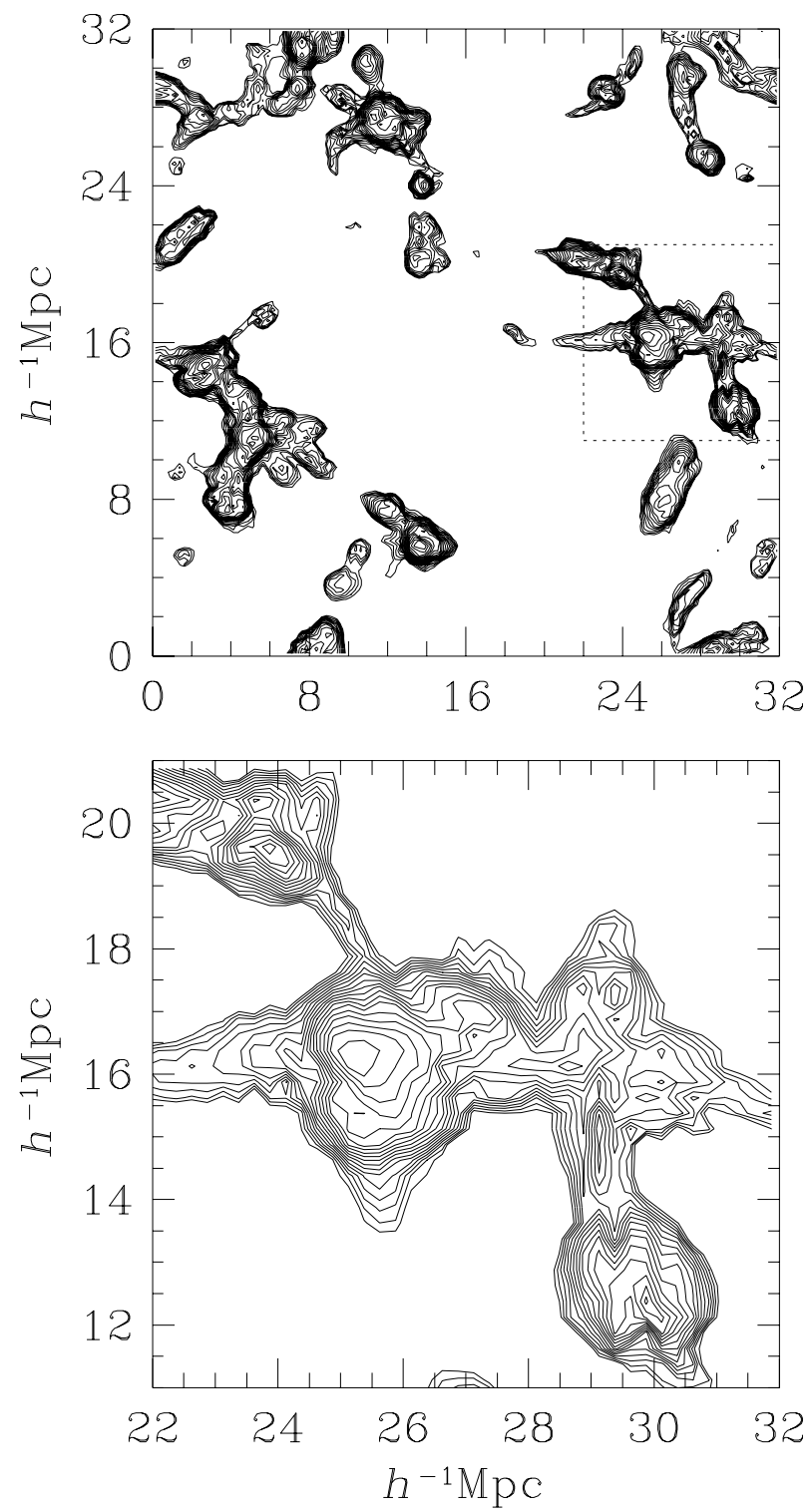

FIG. 3.-Magnetic field strength contours of a slice with a thickness of 2 $h^{-1} \mathrm{Mpc}$ (or 8 cells) at $z=2$. The contour lines with magnetic field strength higher than $8 \times 10^{-23} \mathrm{G}$ are shown with levels $8 \times 10^{-23} \times 10^{k}$ and $k=0,0.1,0.2, \ldots, 2$. The upper panel shows the whole region of $32 \times 32 h^{-1} \mathrm{Mpc}$, while the lower panel shows the magnified region of $10 \times 10 h^{-1} \mathrm{Mpc}$.

prising since the equation for the evolution of $-\omega / 1+\chi$ is identical to that for $\omega_{\mathrm{cyc}}=e \boldsymbol{B} / m_{\mathrm{H}} c$, except for dissipative terms.

By taking the curl of the equation of motion in the form

$$
\frac{\partial v}{\partial t}-v \times(\nabla \times v)+\frac{1}{2} \nabla v^{2}=-\frac{\nabla p}{\rho}+v \nabla^{2} v
$$

where $v$ is the kinematic viscosity, one gets

$$
\frac{\partial \omega}{\partial t}=\nabla \times(v \times \omega)-\frac{\nabla p \times \nabla \rho}{\rho^{2}}+v \nabla^{2} \omega .
$$

Now we see, on comparing equation (7) with equation (4), that if dissipative processes are ignored (conditions well satisfied except during the later stages of the simulation), and if we assume that both $\omega_{\text {cyc }}$ and $\omega$ are initially zero, then we should have

$$
\omega_{\mathrm{cyc}}=-\frac{\omega}{(1+\chi)},
$$

a remarkable result.

It must be appreciated that the $\nabla p \times \nabla \rho$ term is zero until some pressure is generated, since usually $p$ is very small initially in the simulation. The generation of $p$ happens generally in shocks where viscosity is certainly important. It can be argued that the jump in $\omega_{\text {cyc }}$ and $-\omega /(1+\chi)$ across a shock should be equal since, if we could treat equation (7) as valid through the shock, the integral of $\nu \nabla^{2} \omega$ is probably small. Thus, $\omega_{\mathrm{cyc}}$ and $\omega$ satisfy essentially the same equation even in the shock.

A check of the above relation is presented in Figure 4. The magnitudes of these two quantities are displayed on a logarithmic scale. Each point represents the two quantities in each cell. The magnitudes in one among eight neighboring cells were plotted. Here $h=\frac{1}{2}$ was used again. If the relation in equation (8) holds exactly, all these points should lie on the line of unit slope. The deviation for small values is presumably due to the different dissipation rates that are not taken into account in the derivation of this relation. At larger values, the correlation is much better, as is to be expected. The rough agreement of $\omega_{\mathrm{cyc}}$ and $\omega /(1+\chi)$ at least for larger values tends to support the relation in equation (8).

Eventually, viscosity does become important, and $\omega$ tends to saturate in mean square average. However, since the twisting of the magnetic field by the $\nabla \times(v \times B)$ term persists, one expects that $\boldsymbol{B}$ will continue to grow. This fact is supported by $\mathrm{G}$. K. Batchellor's discussion in his early paper (Batchellor 1950). Thus, it is indeed surprising that $\boldsymbol{B}$ seems to saturate at the same time and with the same amplitude as $\omega$ does. Is it a coincidence that numerical resistivity becomes important at the same time that viscosity does?

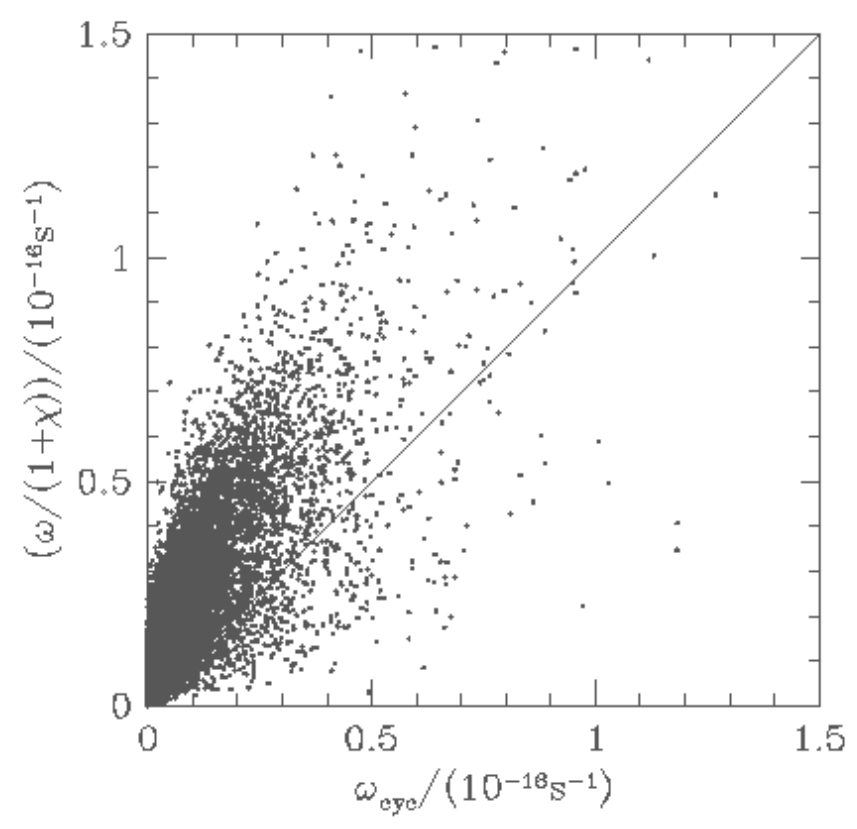

Fig. 4.-Magnitude of $\omega /(1+\chi)$ plotted against that of $\omega_{\text {cyc }}$ on a logarithmic scale. Each point represents the values in each cell. One among eight neighboring cells were plotted. The predicted relation is the $45^{\circ}$ straight line. The correlation is quite good for the larger values. 
Since the effective viscosity is also numerical, this coincidence is probably not so surprising.

It should be noted that the idea that a weak seed magnetic field should be built up during galactic formation was pointed out by Chakrabarti (1991), based on a more qualitative argument than that given here.

\section{ANALYTICAL CALCULATION OF EXPONENTIAL GROWTH OF PROTOGALACTIC MAGNETIC FIELD UP TO SATURATION}

Assuming the saturation of the magnetic field and $\omega$ are numerical, we can argue as follows with respect to what we really think happens to the magnetic field. The value of the numerical viscosity is not much different from the physical viscosity, at least within a few orders of magnitude. But the numerical resistivity is larger than the physical resistivity by great many orders of magnitude. Thus, it would be natural to assume that, in the absence of numerical resistivity and viscosity, the magnetic field should continue to grow, but $\omega$ should not. This supposition is strongly supported by G. K. Batchellor's argument (Batchellor 1950).

To numerically explore the beginnings of the second phase, in which the magnetic field strength grows exponentially, we plan to repeat the simulation under conditions in which the resistivity is smaller than the viscosity. The numerical resistivity and numerical viscosity are fixed by the grid size. However, if an additional viscous term is added to the simulation equations, with the viscosity coefficient clearly larger than the numerical viscosity, then this condition should be achieved. Such a simulation should enable us to confirm that the exponential growth of the magnetic field in phase two is real.

The present numerical simulations have too low a resolution to follow the behavior of the magnetic field into the later phases of evolution, when dynamo action is important. Thus, we turn to the analytical theory of Kulsrud \& Anderson (1992) in order to estimate the amount of growth of the magnetic energy. For this purpose, we assume that during this time, the turbulent motions are represented by Kolmogorov isotropic homogeneous turbulence. The normalization constant in the Kolmogorov spectrum will be determined by the numerical simulation.

Let us examine the three-dimensional power spectra found in the numerical simulations. The numerically determined spectra for $(\nabla \cdot v)^{2}, P_{d}(k)$, and for $(\nabla \times v)^{2}, P_{c}(k)$, at $z=0$, are plotted in Figure 5. At long wavelengths, the amplitude of the perturbations are small, so that linear theory applies. In agreement with the results of Peebles (1993) and others, $P_{c}(k) \rightarrow 0$ as $k \rightarrow 0$, while $P_{d}(k)$ follows the analytic theory expectation, $P_{d}(k) \sim k^{-1}$. Thus, for very long wavenumbers, the $(\nabla \times v)^{2}$ spectrum is very small, while that of the sonic turbulence is large. For these waves, the shear turbulence has not yet gone nonlinear. (Since $\omega$ is driven by the $\nabla p$ term as in equation (7), it cannot really develop until shocks form.)

For wavelengths smaller than about $10 h^{-1} \mathrm{Mpc}$, nonlinearities dominate, and we see that $P_{c}(k) / P_{d}(k) \approx 1$. This spectrum is essentially the shear turbulence spectrum. The corresponding spectrum for sonic turbulence is also given on this plot.

The shear turbulence peaks at a wavenumber of about 1 $\mathrm{Mpc}^{-1}$. For values of $k$ somewhat larger than this peak value, the spectrum follows a power law of $k^{-5 / 3}$. (This

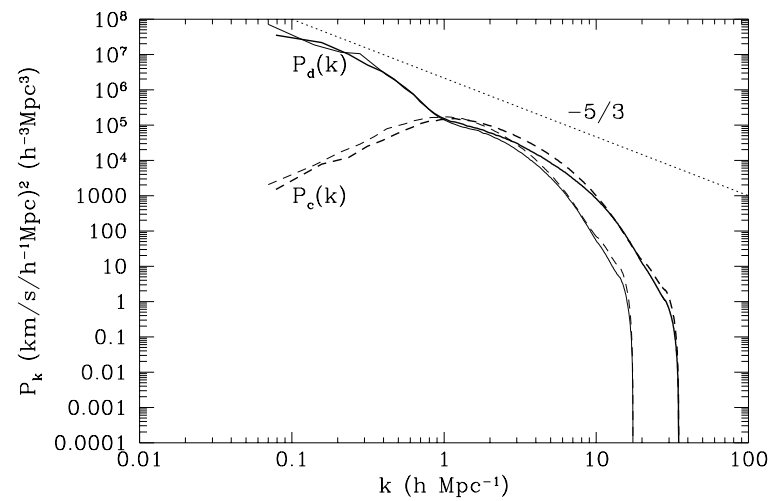

FIG. 5.-Three-dimensional power spectra for $(\nabla \cdot v)^{2}$ (solid curves), $\left\langle(\boldsymbol{\nabla} \cdot \boldsymbol{v})^{2}\right\rangle=\int P_{d}(k) d^{3} \boldsymbol{k}$, and for $(\nabla \times v)^{2}$ (dashed curves), $\left\langle(\nabla \times v)^{2}\right\rangle=$ $\int P_{c}(k) d^{3} k$, produced by numerical simulations. The thick (solid and dashed) curves are from a power-law $\left(P_{k}=k^{-1}\right)$ hydrodynamic simulation with box-size $L=80 h^{-1} \mathrm{Mpc}$ and $512^{3}$ fluid elements, and the thin (solid and dashed) curves are from a power-law $\left(P_{k}=k^{-1}\right)$ hydrodynamic simulation with box-size $L=90 \mathrm{~h}^{-1} \mathrm{Mpc}$ and $288^{3}$ fluid elements. The $L=80$ $h^{-1} \mathrm{Mpc}$ simulation has a resolution half that of the $L=90 h^{-1} \mathrm{Mpc}$ simulation. Comparison of the results from the two simulations indicates that the steep decline at the high- $k$ end of the power spectra is due to limited numerical resolution. A straight dotted line that is parallel to the Kolmogorov spectrum of logarithmic slope $-5 / 3$ is also shown.

power-law behavior for the spectrum is expected for Kolmogorov turbulence. This spectrum should develop from values of $k$ for which the eddies have time to turn over once, since, according to Kolmogorov, this turnover generates a cascade following the Kolmogorov power law. Apparently the eddies at the peak have not yet turned over once, since the power-law spectrum does not extend all the way to the peak. However, at slightly larger wavenumbers, the eddies have apparently turned over, as is indicated by the fact that the Kolmogorov spectrum commences at these $k$.) The $5 / 3$ law does not continue to the smallest scales but falls more rapidly with $k$ as the grid scale is approached. This cutoff is expected for the numerical simulation because of the large numerical viscosity.

To demonstrate the important result that the real turbulent spectrum continues to smaller scales, one can compare it with the spectrum for a finer resolution simulation (see Fig. 5). It is found that the spectrum for the finer resolution extends to smaller scales, as is expected from the smaller effective viscosity if the turbulence is Kolmogorov. This is evidence that, in the real physical situation, there should exist a complete Kolmogorov spectrum extending down to the much smaller true viscous scale.

Although the numerical results are presented at $z=0$, the qualitative behavior should be the same as at earlier values of $z$. We apply the general Kolmogorov theory to earlier epochs, when most galaxies were thought to have formed, and employ similar scalings for its amplitude.

Let us introduce the one-dimensional spectrum for $v^{2}$,

$$
v^{2}=\int I(k) d k
$$

Then, if the turbulence is isotropic, we have

$$
(\nabla \times v)^{2}=4 \pi \int P(k) k^{2} d k=\int k^{2} I d k,
$$

so that $4 \pi P(k)=I(k)$. 
We assume that the Kolmogorov spectrum starts at the peak, $k_{\min }$, and extends down to some maximum $k, k_{\max }$, where it is truncated by physical viscosity. That is, we write the Kolmogorov spectrum as

$$
I=\frac{2}{3} v_{0}^{2} \frac{k_{\min }^{2 / 3}}{k^{5 / 3}},
$$

where $v_{0}^{2}$ is the mean square turbulent velocity.

From the analytic theory for the buildup of magnetic energy given by Kulsrud \& Anderson (1992), we have

$$
\frac{d \mathscr{E}_{M}}{d t}=2 \gamma \mathscr{E}_{M},
$$

where $\mathscr{E}_{M}$ is the magnetic energy; $\gamma$ depends on the Kolmogorov turbulence as

$$
2 \gamma \approx \int \frac{k^{2} I(k)}{\Delta \omega_{k}} d k
$$

where $\Delta \omega_{k}$ is the decorrelation rate at each $k$ size. We take $\Delta \omega_{k}$ to be the eddy turnover rate $k \tilde{v}_{k}$, where $\tilde{v}_{k} \approx[k I(k)]^{1 / 2}$ is the typical eddy velocity at the $k$ scale. Combining these results, we find

$$
2 \gamma=\int \sqrt{k I} d k \approx v_{0} k_{\min }^{1 / 3} \int_{k_{\min }}^{k_{\max }} \frac{d k}{k^{1 / 3}} \approx \frac{3}{2} v_{0} k_{\min }^{1 / 3} k_{\max }^{2 / 3},
$$

if $k_{\max } \gg k_{\min }$. This is essentially the turnover rate of the shortest eddy with wavenumber $k_{\max }$.

Now, for a kinematic viscosity, $v=l v_{\text {th }}$, where $l$ is the mean free path and $v_{\text {th }}$ is the ion thermal velocity, we have

$$
\frac{k_{\max }}{k_{\min }}=R^{3 / 4}=\left(\frac{v_{0}^{2}}{k_{\min } v}\right)^{3 / 4}=\left(\frac{v_{0}^{2}}{v_{\mathrm{th}} k_{\min } l}\right)^{3 / 4} .
$$

Therefore,

$$
\gamma=\frac{3}{4} \frac{v_{0}^{3 / 2}}{v_{\mathrm{th}}^{1 / 2}} \frac{k_{\min }^{1 / 2}}{l^{1 / 2}}
$$

A more accurate value for $\gamma$ is derived in Appendix A. It is

$$
\gamma=1.236 \frac{k_{0}^{1 / 2} v_{0}^{3 / 2}}{l_{0}^{1 / 2} v_{i}^{1 / 2} \sigma \epsilon^{1 / 2}}
$$

In deriving this formula, we set $k_{\min }=k_{0}$ and assume the spectrum is zero for $k<k_{0}$. The mean free path $l_{0}=$ $10^{12} T_{\mathrm{eV}}^{2} / n_{B} \mathrm{~cm}$, and the thermal velocity $v_{i}=10^{6}\left(T_{\mathrm{eV}}\right)^{1 / 2} \mathrm{~cm}$ $\mathrm{s}^{-1} . T_{\mathrm{eV}}$ is the temperature in electron volts. The constants $\sigma$ and $\epsilon$ are of order unity. They are introduced in Appendix $A$ and are related to the model for Kolmogorov turbulence employed there. The quantity $2 \gamma$ is the exponential growth rate of the magnetic energy in a stationary fluid, and $\gamma$ is the growth rate of the rms field strength.

In the expression for $\gamma$, we expect $v_{0}$ to be related to the thermal energy $3 n_{B} k_{\mathrm{B}} T$, where $k_{\mathrm{B}}$ is the Boltzmann constant. Let

$$
\frac{1}{2} \rho v_{0}^{2}=3 \beta n_{B} k_{\mathrm{B}} T,
$$

where $\beta$ is the ratio of turbulent energy to thermal energy. (This ratio should be of order unity since, behind a strong shock, the ratio of kinetic energy to thermal energy is 15/16.) Thus,

$$
\gamma=4.59 \frac{\beta^{3 / 4}}{\sigma \epsilon^{1 / 2}} \frac{k_{0}^{1 / 2} v_{i}}{l_{0}^{1 / 2}}
$$

Let us imagine that the plasma is initially in the form of a sphere of radius $R=2 \pi / k_{0}$ and collapses to form a galaxy. (The collapse is more likely to be two-dimensional, forming an oblate spheroid rather than a sphere. In this case, $R$ should refer to the shorter axis. However, the results on the amplification of the magnetic field should not be very different from those for a spherical collapse.)

The magnetic field is also amplified by the collapse as well as by the turbulence. We assume that the largest turbulent eddy has the same radius as the galaxy, so $k_{0}=2 \pi / R$. Then, if $B$ is the rms value of the magnetic field, we have

$$
\frac{d\left(B R^{2}\right)}{d t}=\gamma B R^{2}
$$

We expect the sphere to collapse by a factor of order 2 in the dynamic time

$$
t_{D}=\frac{1}{\sqrt{4 \pi G n_{B} m_{\mathrm{H}}\left(\rho_{D} / \rho_{B}\right)}}=\frac{8.4 \times 10^{14}}{\sqrt{n_{B}}} \sqrt{\frac{\rho_{B}}{\rho_{D}}} \mathrm{~s},
$$

where $\rho_{D} / \rho_{B}$ is the ratio of dark matter density to baryonic density.

Thus, the amount of amplification during the collapse of the sphere by a single factor of 2 is of order $\gamma t_{D}$. Substituting $k_{0}=2 \pi / R$, and introducing the numerical values for $l_{0}, v_{i}$, and $t_{D}$, we get

$$
\gamma t_{D}=\frac{\beta^{3 / 4}}{\sigma \epsilon^{1 / 2}} \frac{9.67 \times 10^{15}}{\sqrt{R T_{\mathrm{eV}}}} \sqrt{\frac{\rho_{B}}{\rho_{D}}} .
$$

$T_{\mathrm{eV}}$ is the temperature in electron volts.

Now, at the time of collapse, we expect that the temperature $T$ should be comparable to the Jeans's temperature. Therefore, we take

$$
k_{\mathrm{B}} R T_{\mathrm{eV}}=\alpha G M m_{\mathrm{H}}
$$

where $k_{\mathrm{B}}$ is Boltzmann's constant and $M$ is the total mass of the sphere, dark matter plus baryonic matter. Let $M=2$ $\times 10^{44} M_{11} \rho_{D} / \rho_{B} \mathrm{~g}$, where $M_{11}$ is the baryonic mass of the galaxy in units of $10^{11}$ solar masses; $\alpha$ is the ratio of $T$ to the Jeans's temperature and is less than unity. During the collapse, the temperature should remain isothermal because of cooling, or at least it should remain smaller than the Jeans's temperature. For simplicity, we take $\alpha$ to be constant. Its actual effective value should be smaller than unity because of cooling. Thus,

$$
\gamma t_{D}=\frac{2585}{\sqrt{M_{11}}} \frac{\beta^{3 / 4}}{\alpha^{1 / 2} \sigma \epsilon^{1 / 2}}\left(\frac{\rho_{B}}{\rho_{D}}\right) .
$$

To form a more precise estimate of the factor by which $B R^{2}$ grows, we need the integral

$$
A=\int \gamma d t
$$

Let us consider as an example a uniform-density, isolated sphere of radius $R$. Let the initial radius be $R_{0}$, the initial value of $\gamma$ be $\gamma_{0}$, and the initial value of $t_{D}$ be $t_{D 0}$. Let $R=x R_{0}$. During the collapse of the sphere, the pressure of the baryonic matter is negligible, so that the equation for the time dependence of $x$ is

$$
\frac{d^{2} x}{d t^{2}}=-\frac{4 \pi}{3} G \rho_{0} \frac{1}{x^{3}}
$$


where $\rho_{0}$ is the initial density.

Integrating equation (26), we have

$$
\frac{1}{2}\left(\frac{d x}{d t}\right)^{2}=\frac{4 \pi G \rho_{0}}{3} \frac{1}{x}+\text { const } .
$$

For simplicity, we neglect this constant, so that

$$
\frac{d x}{d t}=\sqrt{\frac{2}{3}} \frac{1}{t_{D 0} x^{1 / 2}} .
$$

From equation (24), we have $\gamma \sim t_{D}^{-1} \sim \rho^{1 / 2} \sim x^{-3 / 2}$ as time varies. Using this scaling, we can now integrate $\gamma$ in time

$$
A=\int \gamma d t=\int \gamma \frac{d x}{d x / d t}=\gamma_{0} t_{D 0} \sqrt{\frac{3}{2}} \int \frac{d x}{x} .
$$

Substituting for $\gamma_{0} t_{D 0}$ from equation (24), we have

$$
A=\int \gamma d t=\frac{3166}{\sqrt{M_{11}}} \frac{\rho_{B}}{\rho_{D}} \frac{\beta^{3 / 4}}{\sigma \epsilon^{1 / 2} \alpha^{1 / 2}} \ln \frac{1}{x} .
$$

Taking $\rho_{B} / \rho_{D}=1 / 20$ and $x=1 / 2$, we have

$$
A=\int \gamma d t=\frac{109.7}{\sqrt{M_{11}}} \frac{\beta^{3 / 4}}{\sigma \epsilon^{1 / 2} \alpha^{1 / 2}} .
$$

The number of powers of 10 by which $R^{2} B$ increases during the dynamic time, $A_{10}$, is

$$
A_{10}=\log _{10} e \int \gamma d t=\frac{47.6}{\sqrt{M_{11}}} \frac{\beta^{3 / 4}}{\alpha^{1 / 2} \epsilon^{1 / 2} \sigma} .
$$

\section{EVOLUTION OF MAGNETIC FIELD AFTER SATURATION}

An increase in the rms magnetic field strength by this large number of powers of 10 clearly indicates that $B$ should grow to reach saturation with the turbulent power. Just how the saturation proceeds is somewhat complicated and deserves further analysis. However, from the results of Kulsrud \& Anderson (1992), we can make some remarks as to how we think that the saturation should proceed.

First, before saturation occurs, during the period when the magnetic energy grows exponentially, the wavenumber at which the magnetic energy is concentrated propagates to very small wavelengths. The peak wavenumber $k_{\text {peak }}$ of the magnetic spectrum, $M_{B}(k)$, increases exponentially, as $k_{\text {peak }} \approx e^{\gamma t / 2}$. The magnetic spectrum itself increases as $k^{3 / 2}$ up to $k_{\text {peak }}$ and then falls off rapidly at larger wavenumbers. At any fixed $k$, the magnetic spectrum grows in time as $e^{3 \gamma t / 4}$. The total integrated magnetic energy, $\mathscr{E}_{M}=\int M_{B}(k)$, thus increases as $e^{2 \gamma t}$, as it should.

Second, the magnetic energy at scale $k$ has an Alfvén frequency $\omega_{\mathrm{A}}(k)=k\left(2 \mathscr{E}_{M} / \rho\right)^{1 / 2}$ (Chandran 1997). As $\mathscr{E}_{M}$ increases and $k_{\text {peak }}$ increases, there comes a time when the frequency $\omega_{\mathrm{A}}\left(k_{\text {peak }}\right)$ exceeds the eddy turnover rate $\gamma$. When this happens, the eddy frequency become mismatched with the Alfvén frequency, and amplification of magnetic energy at these scales is no longer possible. This means that for scales such that $k>k_{\text {crit }}$, where

$$
\omega_{\mathrm{A}}\left(k_{\text {crit }}\right)=k_{\text {crit }} \sqrt{2 \mathscr{E}_{M} / \rho}=\gamma,
$$

the magnetic energy no longer grows exponentially. It is seen that as $\mathscr{E}_{M}$ increases, $k_{\text {crit }}$ decreases. It can be shown that, by the time that $k_{\text {crit }}$ becomes as small as $k_{\max }$, the wavenumber of the smallest turbulent eddy, the magnetic energy is concentrated at $k_{\max }$.

Third, at this latter time, $2 \gamma \mathscr{E}_{M}$ is comparable to the turbulent power $\left(\approx k \rho v_{0}^{3} / 2\right)$. After this time, the magnetic energy stops growing exponentially and starts growing linearly in time. This transition happens because the drain on the turbulent power by the magnetic field is stronger than the drain by viscous dissipation. Hence, the hydrodynamic spectrum is truncated by the drain of energy into the magnetic energy rather than by viscous dissipation. As a consequence, $k_{\max }$ decreases at just such a rate that $\gamma$, which depends on $k_{\max }$ as $k_{\max }^{2 / 3}$, decreases so as to keep $2 \gamma \mathscr{E}_{M}$ always equal to the turbulent power. At the time when saturation commences, the cutoff wavenumber $k_{\text {crit }}=k_{\max }$. Subsequently, the wavenumber range in which the magnetic energy is predominant continues to be at $k_{\max }$, and this wavenumber becomes smaller and smaller. In the end, when essentially all the turbulent energy has been converted to magnetic energy, the magnetic energy is all concentrated near $k_{\max } \approx k_{\min }$.

To determine if this last phase is reached, we must ask whether, during phase two, when exponential growth occurs, the magnetic energy at $k_{\max }$ reaches the value $\mathscr{E}_{\text {sat }}$, given

$$
\frac{3}{4} \gamma \mathscr{E}_{\text {sat }}=\frac{1}{2} k_{\min } \rho v_{0}^{3} .
$$

This is the value of magnetic energy at which the turbulent power into the magnetic field is comparable to the total turbulent power. [At this time, the turbulent power at smaller scales has stopped growing. The rate of growth of magnetic energy is $\left(\frac{3}{4}\right) \gamma$ rather than $2 \gamma$.] If this condition is satisfied, then we expect that all the turbulent energy will convert to magnetic energy. Thus, $\mathscr{E}_{\text {sat }}$ is a critical value for $\mathscr{E}_{M}$. At this magnitude and beyond, all the magnetic energy is in a band less than $k_{\max }$. The energy in this limited range has grown only by the factor $e^{3 \gamma t / 4}$. Thus, during collapse of the galaxy, the important number is the number of $e$ foldings of the magnetic energy in the range $k_{0}-k_{\max }$. For total saturation, it must be large enough for $\mathscr{E}_{M}$ to reach this critical value. Since the number of $e$-folds of the energy in this limited range is $\left(\frac{3}{4}\right) \int \gamma d t$, the number of powers of 10 by which the effective value of $B R^{2}$ increases during the collapse is reduced from that of equation (32) by a factor of $3 / 8$, that is, to

$$
A_{10}^{\prime}=\frac{3}{8} A_{10}=\frac{17.87}{\sqrt{M_{11}}} \frac{\beta^{3 / 4}}{\alpha^{1 / 2} \epsilon^{1 / 2} \sigma} .
$$

If this number is large enough to amplify the magnetic field up to the saturated value, such that

$$
\frac{3}{4} \gamma \mathscr{E}_{M}=\frac{1}{2} k_{\min } \rho v_{0}^{3},
$$

then the remaining turbulent energy will be converted to magnetic energy at a linear rate, and the field will become coherent on the scale of the largest turbulent eddy, $k_{\min }^{-1}$. On the other hand, if this number is too small to reach the saturated value of the magnetic field before the turbulence damps, then the resulting magnetic field strength is smaller, and the magnetic field is much more chaotic.

For the determination of the critical number, $A_{10}^{\prime}$, we need values for $\alpha, \beta, \sigma$, and $\epsilon$. For a lower limit on $A_{10}^{\prime}$, we may take $\alpha=1$, since we expect the temperature at the commencement of collapse to certainly be less than the Jeans's temperature $T_{\mathrm{J}}$. A realistic estimate of $\sigma$, where 


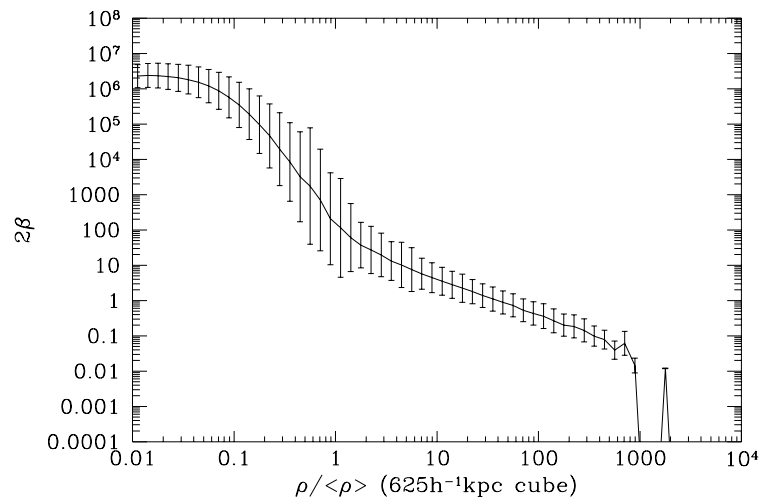

FIG. 6.-Ratio of the local kinetic energy to the thermal energy density in a numerical simulation, as a function of the local relative density $\rho /\langle\rho\rangle$; $\langle\rho\rangle$ is the mean density. If the turbulent kinetic energy density is one-half of the total kinetic energy density, then this ratio is equal to $2 \beta$, where $\beta$ is the normalizing parameter introduced in the text as the ratio of turbulent energy density to thermal energy density.

$\sigma \Delta \omega_{k}$ is the breadth frequency spectrum of the turbulence at fixed $k$, is about 2. (The definitions of these parameters are given just before eq. [A4].) A value of $\epsilon$ of 1 seems plausible where the parameter $\epsilon$ is related to the rate of turbulent transfer (see eq. [A14]). To find $\beta$, we make use of the numerical simulation; $\beta$ is the ratio of turbulent kinetic energy to thermal energy. If we assume that the turbulent energy is one-half of the total kinetic energy, then we can take the ratio of total kinetic energy density to thermal energy to be $2 \beta$. This ratio depends on the ratio of the density to the mean density, $\rho /\langle\rho\rangle$. Its value, taken from the numerical simulation, is plotted in Figure 6. The appropriate value for $\rho /\langle\rho\rangle$ at the time of collapse is 5 (Peebles 1993). From Figure 6, we find from this value for the relative density that $\beta \approx 5$. Thus, substituting $\alpha=1, \beta=5, \sigma=2$, and $\epsilon=1$ into equation (35), we find that the number of powers of 10 by which $B$ increases is

$$
A_{10}^{\prime}=30 \text {. }
$$

This number is certainly sufficiently large to show that $B$ increases, from the value $10^{-21} \mathrm{G}$ obtained from the Biermann battery mechanism, to the critical value of $B$ for saturation with the turbulent power. (For the value of $\gamma$ found from eq. [24] with our choice of parameters, this critical field strength for $B$ is about $10^{-7} \mathrm{G}$. If this critical value for the magnetic field strength is reached, then we expect equipartition with the total turbulent energy to result. This final value of $B$ is about $10^{-5} \mathrm{G}$.)

\section{CONCLUSION}

In conclusion, we have described a mechanism for generating the observed cosmic magnetic fields that proceeds in three phases.

In the first phase, the Biermann battery mechanism, driven by cosmic turbulence originating in shocks and caustic formation, creates a magnetic field from a zero initial field. This mechanism is found to produce a magnetic field whose strength is about $10^{-21} \mathrm{G}$. In the second phase, Kolmogorov turbulence amplifies this field by a factor sufficient to reach saturation with the turbulent power. (Neglecting saturation, the factor of increase would be of order $10^{30}$.) In the third phase, the magnetic field reaches equipartition with the turbulent energy, yielding a field strength of order $10^{-5} \mathrm{G}$. During this phase, it becomes coherent on the scale of the largest turbulent eddy. This scale is comparable to the scale of the entire galaxy.

The final value of the magnetic field, which we predict to be in equipartition with the turbulent energy, is very strong. In fact, it is too strong to allow interstellar matter to flatten into a disk once it separates from the dark matter halo. The likely state of affairs at this time is that the sphere of interstellar plasma cannot immediately collapse to a disk. Probably, what happens is that star formation occurs, removing a substantial fraction of the interstellar plasma from the magnetic field. The remnant of the plasma is then too light to hold the magnetic field, and the bulk of the remaining plasma is expelled back into the intergalactic or intracluster medium, carrying the field with it. The remaining field can then be compressed by the interstellar medium and enriched by matter thrown off by the evolved halo stars. Such a process should leave some observational trace, which might be used to check this theory of the protogalactic origin of the cosmic field.

It should be remarked that the dynamo action that we have examined has no $\alpha$-effect such as is usually postulated for mean field dynamo theory. These theories are kinematic and ignore any effect of magnetic forces. Thus, to produce a coherent field in the kinematic limit, some coherence in the turbulence is necessary. However, when the field becomes very strong, it generates its own coherence, since it is impossible to put a large energy into small scales. This is because the magnetic tension would become too strong if the magnetic field strength is increased on small scales. The magnetic field is perfectly capable of unwinding itself, and producing its own coherence, when it is sufficiently strong. The necessity for an $\alpha$-effect is purely a weak field result, only applicable in the kinematic limit.

It is interesting that the triple-phase process of creation of cosmic magnetic fields was suggested by L. Biermann himself many years ago (Biermann 1950).

The authors are grateful for a helpful discussion with A. Achterburg, Ben Chandran, Steven Cowley, Ravi Sudan, Martin Rees, and Ethan Vishniac. The work of R. M. Kulsrud was supported by the National Science Foundation under grant AST 91-21847 and by NASA's astrophysical program under grant NAGW 2419. The work by D. Ryu was supported in part by the Basic Science Research Institute Program, Korean Ministry of Education 1995, Project No. BSRI-95-5408. The work by R. Cen and J. P. Ostriker was supported by the National Science Foundation grant AST 93-18185.

\section{APPENDIX A}

\section{A MORE PRECISE VALUE FOR GROWTH RATE $\gamma$}

In this appendix, we evaluate the growth rate of the rms magnetic field for the Kolmogorov spectrum of the turbulent energy per unit mass per unit wavenumber, $I(k) . I(k)$ is the one-dimensional spectrum defined in equation (9). We assume that $I$ is zero for $k<k_{0}$, and in the inertial range $k \ll k_{\max }$, where $k_{\max }$ is the cutoff wavenumber, $I(k)$ is given by 


$$
I(k)=\frac{2}{3} v_{0}^{2} \frac{k_{0}^{2 / 3}}{k^{5 / 3}} .
$$

Here $v_{0}^{2}$ is the mean square value of the turbulent velocity. $I(k)$ suffers a viscous cutoff at $k_{\max }$. We make use of the formalism of Kulsrud \& Anderson (1992).

In Kulsrud \& Anderson, $v_{0}^{2} / 2$ is given in terms of the $k, \omega$ spectrum, $J(k, \omega)$, the energy per wavenumber mode, per unit angular frequency, per unit mass in a box normalization of size $L$,

$$
\frac{v_{0}^{2}}{2}=\left(\frac{L}{2 \pi}\right)^{3} \int d^{3} k d \omega J(k, \omega)
$$

while $\gamma$ is expressed in terms of the quantity

$$
U(k)=\left(\frac{L}{2 \pi}\right)^{3} 2 \pi J(k, 0),
$$

which involves the zero-frequency harmonic of the turbulence.

We assume the frequency spectrum at each $k$ has a width $\sigma(\Delta \omega)_{k}$, where

$$
(\Delta \omega)_{k}=k \tilde{v}_{k}
$$

and $\sigma$ is a constant of order unity. Here

$$
\tilde{v}_{k}^{2}=\int_{k / 2}^{2 k} I(k) d k
$$

is the contribution from a range about $k$ extending from $k / 2$ to $2 k$ :

$$
\tilde{v}_{k}^{2}=\frac{3}{2} k I\left[2^{2 / 3}-\left(\frac{1}{2}\right)^{2 / 3}\right]=1.436 k I
$$

so that

$$
(\Delta \omega)_{k}=1.198 k^{3 / 2} I^{1 / 2}
$$

Thus, we have

$$
\int J(k, \omega) d \omega=J(k, 0) \sigma(\Delta \omega)_{k}
$$

Combining the above equation with equations (A2) and (A3) and the definition of $I(k)$, we get

$$
U(k)=\frac{I(k)}{4 k^{2} \sigma(\Delta \omega)_{k}} .
$$

Substituting equation (A7) in this equation, we get

$$
U(k)=0.2086 \frac{I(k)^{1 / 2}}{\sigma k^{7 / 2}} .
$$

Now, in the kinematic limit, according to Kulsrud \& Anderson (1992), the total magnetic energy $\mathscr{E}_{M}$ grows at the rate

$$
\frac{d \mathscr{E}_{M}}{d t}=2 \gamma \mathscr{E}_{M}
$$

where, according to their equation (2.44),

$$
\gamma=\frac{1}{3} \int_{k_{0}}^{k_{\max }} k^{2} U d^{3} k=\frac{4 \pi}{3} \int_{k_{0}}^{k_{\max }} k^{4} U d k
$$

Substituting equation (A10) for $U$, we get

$$
\gamma=0.8738 \int_{k_{0}}^{k_{\max }} \frac{(k I)^{1 / 2}}{\sigma} d k
$$

The Kolmogorov spectrum $I(k)$ is given for $k$ not too near $k_{\max }$ by equation (A1). In order to find a more complete expression for $I$ near $k_{\max }$, we carry out a dimensional analysis of the Kolmogorov spectrum.

We assume that, due to mode coupling, all the energy from a band $k / 2-k$ is moved to the band $k-2 k$ in a time $1 / \epsilon(\Delta \omega)_{k}$. Here $\epsilon$ is a constant of order unity. Thus, the flux of energy passing through $k, \Phi$, is

$$
\Phi=\frac{1}{2} \epsilon(\Delta \omega)_{k} \int_{k / 2}^{k} I d k=\epsilon(\Delta \omega)_{k} \frac{3}{4}\left(2^{2 / 3}-1\right) k I=0.4406 \epsilon(\Delta \omega)_{k} k I
$$


In the evaluation of the constant, we have employed the power-law approximation for the inertial range of $I(k)$, equation (A1).

Now, according to Braginskii (1965), energy is dissipated by viscosity at the rate

$$
\eta\left[2\left(\frac{\partial v_{z}}{\partial z}\right)^{2}+\left(\frac{\partial v_{x}}{\partial x}\right)^{2}+\left(\frac{\partial v_{y}}{\partial y}\right)^{2}\right]
$$

where $z$ is the prevailing direction of the rms magnetic field. The above result is correct as long as the gyroradius is small. Averaging this result over all directions, the energy dissipated per unit $k$ is

$$
\frac{\eta k v^{2}}{5}=\frac{\eta k^{2} I}{5}
$$

Thus, equating the derivative of the flux $\Phi$ to this viscous damping rate, we get the approximate equation for $I$,

$$
0.4408 \frac{\partial}{\partial k}\left[\epsilon(\Delta \omega)_{k} k I\right]=-\frac{\eta k^{2} I}{5} .
$$

The solution to this equation that agrees with equation (A1) is

$$
I(k)=\frac{2}{3} \frac{v_{0}^{2} k_{0}^{2 / 3}}{k^{5 / 3}}\left[1-\left(\frac{k}{k_{\max }}\right)^{4 / 3}\right],
$$

where

$$
k_{\max }=5.033 \frac{v_{0}^{3 / 4} k_{0}^{1 / 4}}{\eta^{3 / 4}} .
$$

Substituting equation (A18) into equation (A13), we get

$$
\gamma=\frac{4 \pi}{18 \sigma} k_{0}^{1 / 3} k_{\max }^{2 / 3} v_{0}
$$

Furthermore, substituting equation (A19) for $k_{\max }$, we get

$$
\gamma=2.050 \frac{v_{0}^{3 / 2} k_{0}^{1 / 2}}{\sigma \epsilon^{1 / 2} \eta^{1 / 2}}
$$

As given by Braginskii, the ion viscosity can be expressed as

$$
\eta=2.75 v_{i} l_{0}
$$

where $v_{i}$ is the ion thermal velocity and $l_{0}$ is the ion mean free path given by

$$
\begin{aligned}
& v_{i}=10^{6} T_{\mathrm{eV}} \mathrm{cm} \mathrm{s}^{-1}, \\
& l_{0}=10^{12} T_{\mathrm{eV}}^{2} / n_{B} \mathrm{~cm} .
\end{aligned}
$$

Here $T_{\mathrm{eV}}$ is the ion temperature expressed in electron volts, and $n_{B}$ is the ion density. Substituting equation (A22) into equation (A21), we get

$$
\gamma=1.236 \frac{v_{0}^{3 / 2} k_{0}^{1 / 2}}{\sigma \epsilon^{1 / 2} v_{i}^{1 / 2} l_{0}^{1 / 2}}
$$

which is equation (17).

\section{REFERENCES}

Bardeen, J. M., Bond, J. R., Kaiser, N., \& Szalay, A. S. 1986, ApJ, 304, 15

Batchellor, G. K. 1950, Proc. R. Soc. London A, 201, 405

Biermann, L. 1950, Z. Naturforsch, 5a, 65

Braginskii, S. I. 1965, Rev. Plasma Phys., 1, 205

Chakrabarti, S. K. 1991, MNRAS, 252, 246

Chandran, B. 1996, Ph.D. dissertation, Dept. Astrophys. Sci.

. 1997, ApJ, submitted

Harten, A. 1983, J. Comput. Phys., 49, 357
Kang, H., Cen, R., Ostriker, J. P., \& Ryu, D. 1994, ApJ, 428, 1

Kulsrud, R. M., \& Anderson, S. W. 1992, ApJ, 396, 606

Peebles, P. J. E. 1993, Principles of Physical Cosmology (Princeton: Princeton Univ. Press)

Ruzmaikin, A. A., Shukurov, A. M., \& Sokoloff, D. D. 1988, in Astrophysics and Space Science Library, Magnetic Fields in Galaxies (Dordrecht: Kluwer)

Ryu, D., Ostriker, J. P., Kang, H., \& Cen, R. 1993, ApJ, 414, 1 